\title{
Signal transduction mechanism of the NZW type IL-10R1 and the effect on B lymphocyte function
}

\author{
CHENG DIAO $^{1}$, ZANMEI QI $^{2}$, SHUYAN DU $^{1}$, XINZHE CAI $^{1}$ and YI JIANG $^{3}$ \\ ${ }^{1}$ Central Laboratory, The First Affiliated Hospital; ${ }^{2}$ Department of Immunology, College of Basic Medical Sciences; \\ ${ }^{3}$ Central Laboratory and Department of Dermatology, The First Affiliated Hospital, \\ China Medical University, Shengyang, Liaoning 110001, P.R. China
}

Received August 5, 2013; Accepted March 5, 2014

DOI: $10.3892 / \mathrm{mmr} .2014 .2147$

\begin{abstract}
The present study aimed to investigate the signal transduction mechanism of the NZW type interleukin(IL)-10R1 and the effect on the function of B lymphocytes. Vectors for the expression of wild-type, NZW and G1146A IL-10R1 were constructed and transfected into $\mathrm{Ba} / \mathrm{F} 3$ cells. The cell proliferation curve was determined using an MTT assay. CD32 and leukocyte-endothelial cell adhesion molecule 1 (LECAM-1) expression levels were assessed using flow cytometry. The phosphorylation of Janus kinase 1 (Jak1), tyrosine kinase 2 (Tyk2) and signal transducer and activator of transcription 3 (Stat3) were measured by western blot analysis. As compared with wild-type IL-10R1 cells, the proliferation ability of NZW IL-10R1 cells was increased following stimulation with IL-10, which was even greater for G1146A cells. CD32 levels in NZW IL-10R1 cells were lower, while the expression of LECAM-1 was higher compared with wild-type IL-10R1 cells, which was also observed for G1146A cells. The phosphorylation levels of Jak1 and Stat3 in NZW IL-10R1 and G1146A IL-10R1 cells were significantly lower compared with the wild-type IL-10R1 cells. However, the phosphorylation levels in the three cell types were not significantly different. In conclusion, the deficiency in Jak1-Stat3 signal transduction in NZW IL-10R1 cells induces a loss in the inhibition ability of proliferation and activation as well as a migration tendency of B lymphocytes, which is hypothesized to be associated with the occurrence and development of the autoimmune disease systemic lupus erythematosus (SLE).
\end{abstract}

Correspondence to: Dr Yi Jiang, Central Laboratory and Department of Dermatology, The First Affiliated Hospital, China Medical University, 155 Nan-Jing Bei, Shenyang, Liaoning 110001, P.R. China

E-mail: jiangyi_cmu@163.com

Key words: systemic lupus erythematosus, interleukin-10, interleukin-10R1, B lymphocyte, NZW mouse

\section{Introduction}

Systemic lupus erythematosus (SLE) is a typical systemic autoimmune disease (1). The pathogenesis and severity of SLE are associated with the hereditary factor. Abnormalities of the immune system have a role in the occurrence of this disease, which affects almost all monocytes and a number of cytokines. Previous studies have shown that interleukin (IL)-10 has an important role in the pathogenesis of SLE (2-4). The mechanism may be associated with the hypothesis that IL-10 induces immune dysfunction and loss of immunotolerance, and increases the production of antibodies implicated in organ damage (5).

As an inhibitive cytokine, IL-10 is produced by a number of cells. Previous studies have indicated that the regulatory T lymphocyte (Treg) and regulatory B lymphocyte (Breg) are the primary origin of IL-10 in the body (6-8). As with other cytokines, IL-10 is required to bind to the IL-10 receptor (IL-10R) prior to activation. Human and mouse IL-10R, which are members of the interferon receptor family, are composed of two subunits, IL-10R1 and IL-10R2 $(9,10)$. IL-10R2 is considered to be a member of the interferon (IFN) receptor, does not bind to IL-10 and initiates intracellular transduction signals. IL-10R1 has a significant role in IL-10R binding to IL-10. Following binding to IL-10, IL-10R1 transfers the excitability and inhibitory signal through the Janus kinase (Jak)-signal transducer and activator of transcription (Stat) signal transduction system (11). Previous studies suggested that the distribution of the functional regions of IL-10R is located in the cytoplasm (12).

In our previous study, the results of DNA sequencing analysis of the IL-10R1 gene coding region indicated that, compared with the C57BL/6 mouse, the New Zealand W (NZW) mouse has 18 base displacements, among which, 7 regions are hypothesized to cause an amino acid change. Notably, in the no. 1146 base of IL-10R1 of the NZW mouse, a $\mathrm{G}$ was replaced with $\mathrm{A}(\mathrm{G} 1146 \mathrm{~A})$, which induces the change of a coding amino acid from $\mathrm{G}$ to $\mathrm{E}(\mathrm{G} 356 \mathrm{E})$ and the loss of the Jak1 and tyrosine kinase 2 (Tyk2) phosphorylation binding sequence of IL-10R1 (13). G356E is hypothesized to be located at the 298-405 region of the IL-10R1 cytoplasm region, which is responsible for the negative signal transduction of IL-10. Notably, the IL-10R1 gene type of the Murphy Roths large (MRL) mouse, another type of typical SLE model mouse, is 

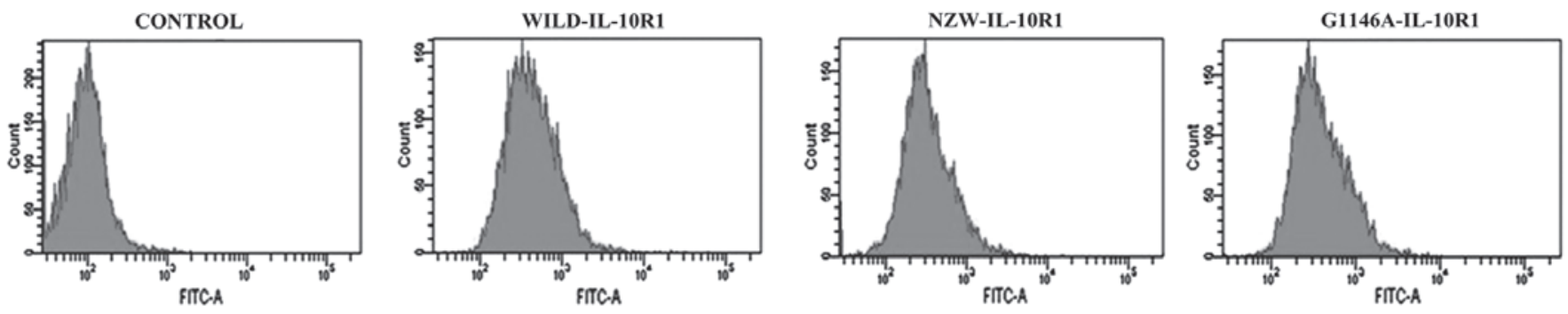

Figure 1. Expression levels of NZW, G1146A and wild-type of IL-10R1 measured by flow cytometry. IL-10, interleukin-10.

A

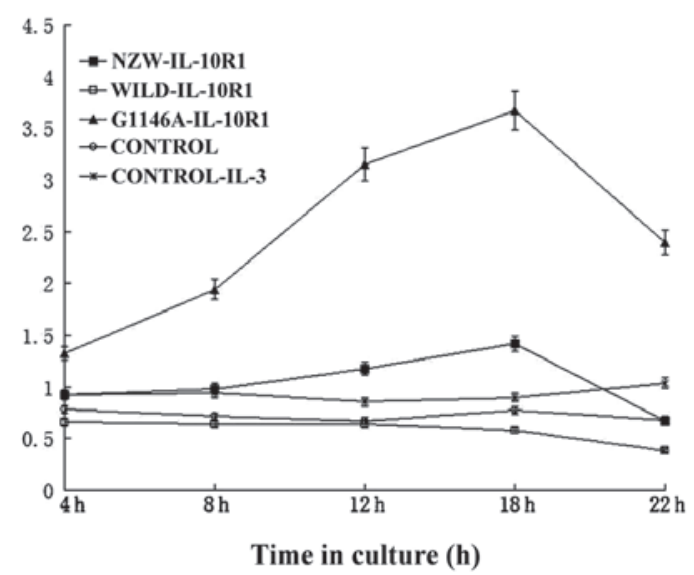

B

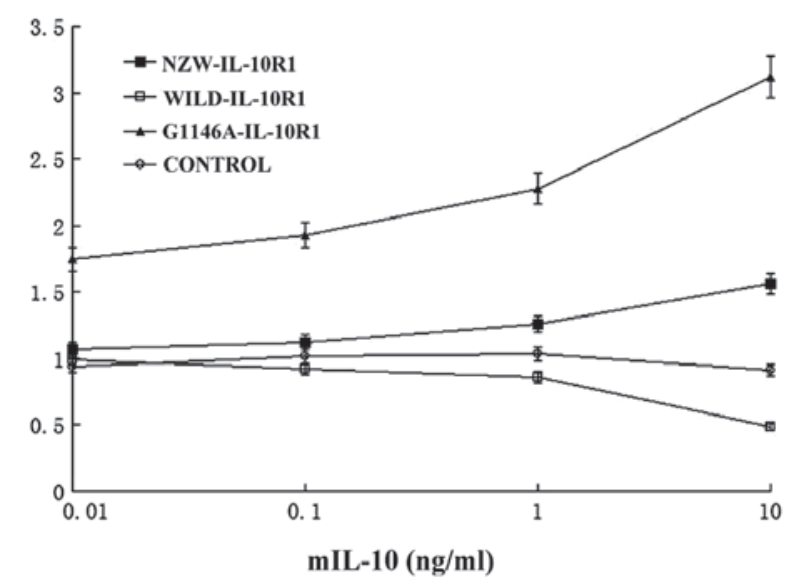

Figure 2. (A) Proliferation curves showed that $1 \mathrm{ng} / \mathrm{ml}$ IL-10 stimulated the growth of BA/F3 cells expressing G1146A-IL-10R1, NZW-IL-10R1 and WILD-IL-10R1 during incubation for 4, 8, 12, 18 and $22 \mathrm{~h}(\mathrm{n}=6)$. (B) Proliferation curves showed that different doses of IL-10 (0.01, 0.1, 1.0 and 10 ng/ml) stimulated the growth of BA/F3 cells expressing G1146A-IL-10R1, NZW-IL-10R1 and WILD-IL-10R1 over 12 h (n=6). IL, interleukin; WILD, wild-type.

the same as the NZE mouse, thus indicating that the defect of IL-10R1 may cause SLE (14). The purpose of the present study was to investigate the signal transduction mechanism of the NZW type IL-10R1 and the effect on the function of B lymphocytes.

\section{Materials and methods}

Cell culture. BA/F3 cells were purchased from the cell center of Peking Union Medical College (Beijing, China). The cells were cultured in Dulbecco's modified Eagle medium (DMEM; Invitrogen, Carlsbad, CA, USA) with $10 \mathrm{ng} / \mathrm{ml} \mathrm{rmIL}-3$ (R\&D Systems, San Diego, CA, USA) and 10\% fetal calf serum (FCS; Invitrogen), and were incubated.

Vector construction. The study was approved by the Ethics Committee of China Medical University (Shenyang, China). Total RNA was extracted from the spleen of C57BL/6 and NZW mice (purchased from Shanghai Laboratory Animal Center of the Chinese Academy of Sciences and maintained under specific pathogen-free conditions). The following primers were designed by Takara Biotechnology (Dalian, China; forward, 5'-GGATCCACC ATGTTGTCGCGTTTGCTCCC-3' and reverse, 5'-CTC GAGTCATTCTTCTACCTGCAG GCTGGAG-3') were used to obtain the coding sequence region of IL-10. DNA was cloned into the pMD19-T simple vector and the DNA sequences were checked using agarose gel electrophoresis. Using GeneBank to compare the results, it was observed that the genes analyzed were not the target genes. Thus, the genes were cloned into the pcDNA3.1(+) multiple clonal sites to produce pcDNA3.1(+)-wild-type (WILD)-IL-10R1 and pcDNA3.1(+)-NZW-IL-10R1. The designed primers (forward, 5'-GAGTCTCCAGAGCTACAGGCCACCTG-3' and reverse, 5'-GCCTCTAGCTCTGGAGACTCTTCTTTTCC-3') were used to perform the G1146A point mutation in pcDNA3.1(+)-WILD-IL-10R1. pcDNA3.1(+)-G1146A-IL-10R1 was confirmed by sequencing using agarose gel electrophoresis.

Transfection. BA/F3 cells $\left(1 \times 10^{5} /\right.$ well) were incubated in 24-well plates for one day prior to transfection. According to the manufacturer's instruction for Lipofectamine 2000 (Invitrogen), pcDNA3.1(+)-WILD-IL-10R1, pcDNA3.1(+)-NZW-IL-10R1 and pcDNA3.1(+)-G1146A-IL-10R1 were transfected into the cells. On the second day following transfection, $500 \mu \mathrm{g} / \mathrm{ml}$ G418 (Gibco, Grand Island, NY, USA) was added to the culture medium. On the fourth day following transfection, culture medium containing $200 \mu \mathrm{g} / \mathrm{ml} \mathrm{G} 418$ was used to culture the cells for subsequent use.

Flow cytometric analysis. Phosphate-buffered saline (PBS) (pH 7.2-7.4) was used with $1 \%$ bovine serum albumin to wash the cells three times and cells were left in $0.5 \mathrm{ml}$ PBS following 
A

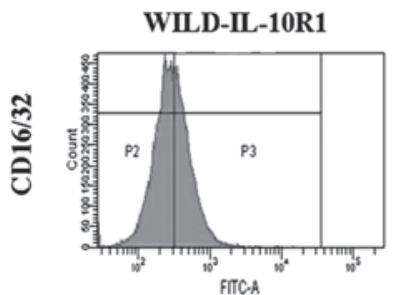

NZW-IL-10R1

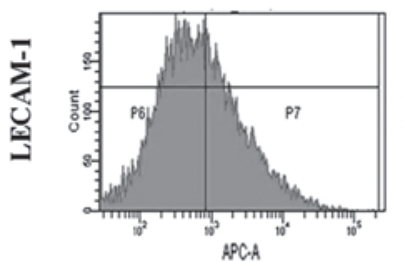

B

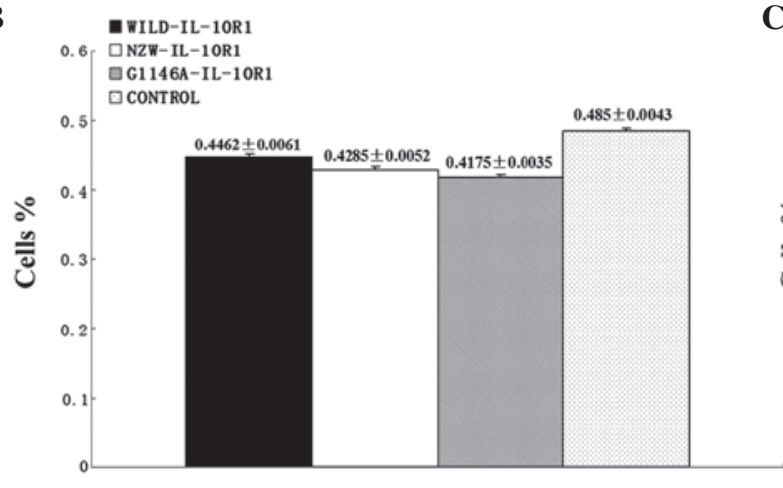

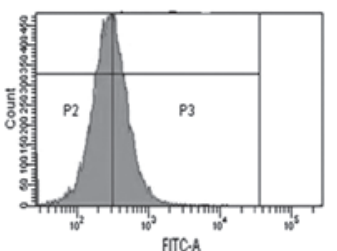

G1146A-IL-10R1
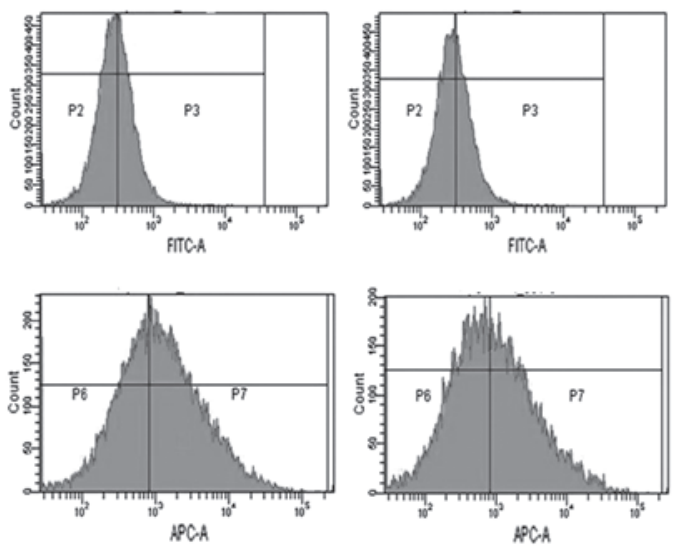

C
$0.7 \begin{aligned} & \text { M WILD-IL-10R1 } \\ & \square \text { NZW-IL-10R1 } \\ & \text { घG1146A-IL-10R }\end{aligned}$

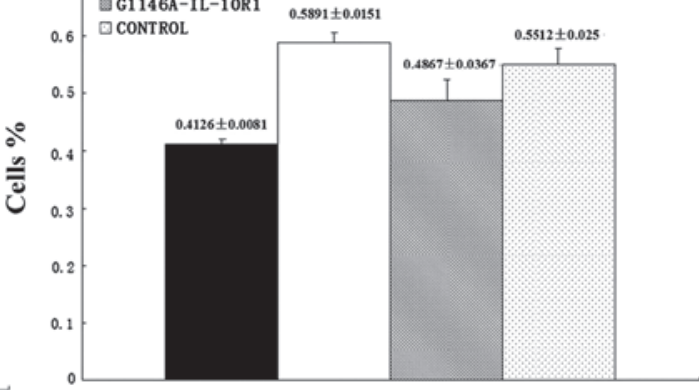

LECAM-1
CONTROL
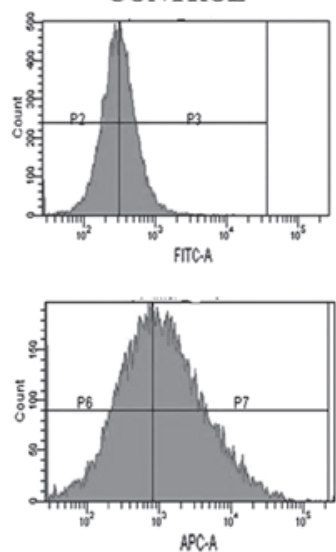

Figure 3. (A) CD16/32 and LECAM-1 expression in BA/F3 cells expressing G1146A-IL-10R1, NZW-IL-10R1 and WILD-IL-10R1 following stimulation with IL-10. (B) Statistical bar chart of the expression of CD16/32 in BA/F3 cells expressing G1146A-IL-10R1, NZW-IL-10R1 and WILD-IL-10R1 following stimulation with IL-10 (P<0.05, n=6). (C) Statistical bar chart of the expression of LECAM-1 in BA/F3 cells expressing G1146A-IL-10R1, NZW-IL-10R1 and WILD-IL-10R1 following stimulation with IL-10 ( $\mathrm{P}<0.05 ; \mathrm{n}=6)$. IL, interleukin; LECAM-1, leukocyte-endothelial cell adhesion molecule 1; WILD, wild-type.

washing. Next, phycoerythrin anti-mouse IL-10R, fluorescein isothiocyanate anti-mouse CD16/32 and allophycocyanin anti-mouse CD62L (BioLegend Inc., San Diego, CA, USA) was added and the cells were incubated at room temperature for $30 \mathrm{~min}$ in the dark. Next, the flow cytometer FACScan (BD Biosciences, San Jose, CA, USA) was used to measure the expression levels of IL-10R1, CD16/32 and CD62L. The results were analyzed by WinMDI 2.9 (The Scripps Research Institute, San Diego, CA, USA).

MTT assay. Cells (1,000 cells/well) were incubated in a 96-well plate with $100 \mu \mathrm{l}$ culture medium. Different concentrations of IL-10 $(0,0.01,0.1,1$ and $10 \mathrm{ng} / \mathrm{ml})$ were used to activate the cells for $12 \mathrm{~h}$. A total of $1 \mathrm{ng} / \mathrm{ml}$ IL-10 was used to activate the cells for different times $(0,4,8,12,18$ and $22 \mathrm{~h})$. Next, $5 \mathrm{mg} / \mathrm{ml}$ MTT (10 $\mu \mathrm{l}$ per well) was added and cells were allowed to incubate at $37^{\circ} \mathrm{C}$ under $5 \% \mathrm{CO}_{2}$ for $3 \mathrm{~h}$. Following the addition of dimethylsulfoxide ( $100 \mu \mathrm{l} /$ well), the plate was agitated for $10 \mathrm{~min}$ and a CLARIOstar microplate reader (BMG Labtech, Ortenberg, Germany) was used to read the optical density value $(490 \mathrm{~nm})$.

Western blot analysis. The cells were starved in DMEM without serum for $12 \mathrm{~h}$, incubated with $10 \mathrm{ng} / \mathrm{ml} \mathrm{IL-10}$ for $15 \mathrm{~min}$ and lysed. Protein solutes $(70 \mu \mathrm{g})$ were purified using $7 \%$ SDS-PAGE. Next, the protein was transferred to a Hybond-enhanced chemiluminescence membrane.

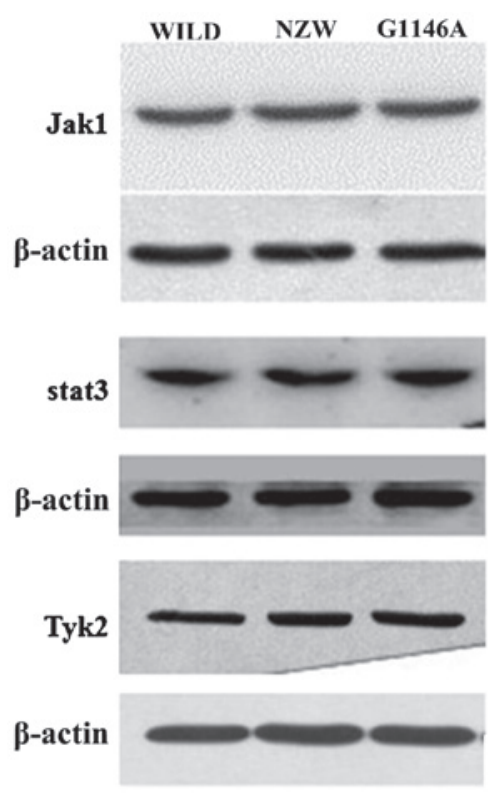

Figure 4. The JAK1, STAT3 and TYK2 levels in three cell types without stimulation ( $\mathrm{n}=4$ ). JAK1, Janus kinase 1; STAT3, signal transducer and activator of transcription 3; TYK2, tyrosine kinase 2 .

The membrane was then blocked for at least $1 \mathrm{~h}$ in $5 \%$ skimmed milk. The membrane was incubated with anti-Jak1, p-Jak1, STAT3, p-STAT3, Tyk2, p-Tyk2 or $\beta$-actin primary 
A
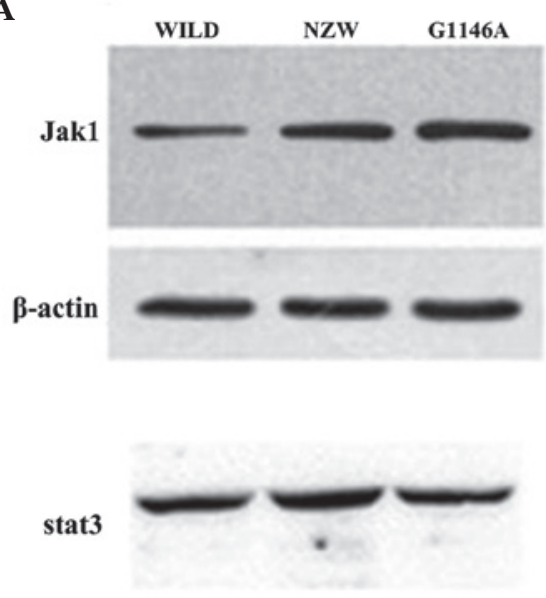

B-actin

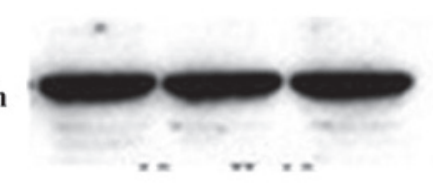

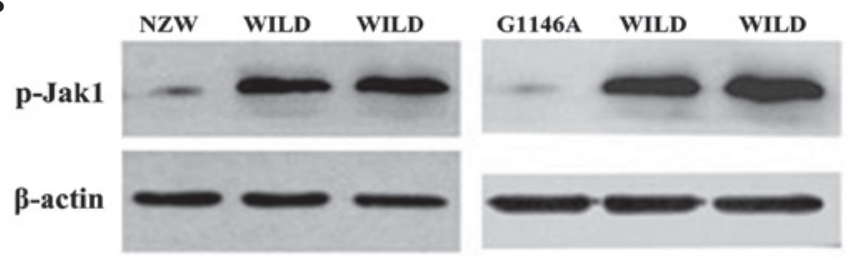

C

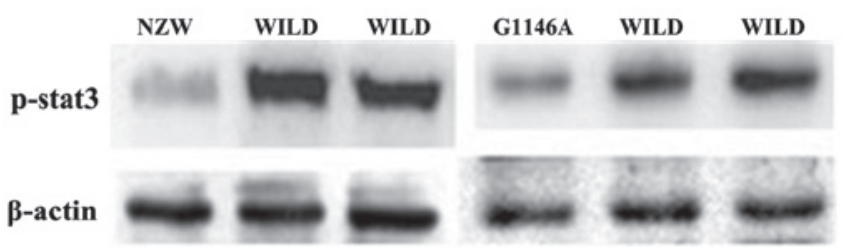

D

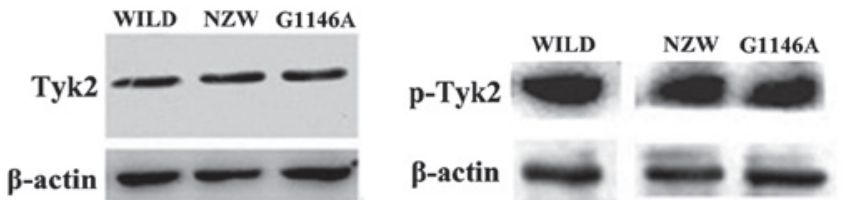

Figure 5. (A) JAK1, STAT3 and TYK2 levels in the three types cells with $10 \mathrm{ng} / \mathrm{ml}$ IL-10 stimulation (n=4). (B) p-JAK1 levels in the three cell types with $10 \mathrm{ng} / \mathrm{ml}$ IL-10 stimulation (n=4). (C) p-STAT3 levels in the three cell types with $10 \mathrm{ng} / \mathrm{ml}$ IL-10 stimulation (n=4). (D) p-TYK2 levels in the three cell types with $10 \mathrm{ng} / \mathrm{ml}$ IL-10 stimulation (n=4). NZW, NZW-IL-10R1; Wild, WILD-IL-10R1; Single, G1146A-IL-10R1; IL, interleukin; p-JAK1, phosphorylated Janus kinase 1; STAT3, signal transducer and activator of transcription 3; TYK2, tyrosine kinase 2; WILD, wild-type.

antibodies (Invitrogen) at $4^{\circ} \mathrm{C}$ overnight. Following washing, the membrane was incubated with the secondary antibodies for $1 \mathrm{~h}$ in the dark. Images were scanned using a Typhoon 9400 scanner (GE Healthcare, Fairfield, CT, USA ).

Statistical analysis. Data are presented as the mean \pm standard error of the mean of at least three independent experiments. For statistical analysis, a one-way analysis of variance was used for comparison of one variance (ANOVA) among groups and two-way ANOVA was used for comparison of two independent variances among groups followed by Tukey's post-hoc test. $\mathrm{P}<0.05$ was considered to indicate a statistically significant difference.

\section{Results}

Transfected cell lines express IL-10R1. The expression levels of IL-10R1 on the surface of BA/F3 cells stably transfected with NZW, G1146 and WILD IL-10R1 expression vectors, respectively, were assessed by flow cytometry and are presented in Fig. 1. The moderately expressing cells were selected for subsequent usage.

IL-10 affects the proliferation of BA/F3 cells expressing different types of IL-10R1. IL-10 was capable of inducing as well as regulating B-cell proliferation. The same dose of IL-10 (1 ng/ml) was used to stimulate the BA/F3 cells expressing G1146A-IL-10R1, NZW-IL-10R1 and WILD-IL-10R1. The BA/F3 cells expressing G1146A-IL-10R1 and NZW-IL-10R1 did not exhibit the ability to inhibit proliferation with progressing stimulation time. However, IL-10 exhibited an ability to induce proliferation. The proliferation curve was steeper compared with the groups without (control cells) and with IL-3. Notably, the proliferation of BA/F3 cells expressing G1146A-IL-10R1 was significantly higher compared with the other groups. IL-10 inhibited the proliferation of the BA/F3 cells expressing WILD-IL-10R1 as the stimulation time was extended. The proliferation curve was markedly lower compared with the group without (control cells) and with IL-3 (Fig. 2A). Different doses of IL-10 $(0.01,0.1,1.0$ and $10 \mathrm{ng} / \mathrm{ml})$ were used to stimulate the BA/F3 cells expressing G1146A-IL-10R1, NZW-IL-10R1 and WILD-IL-10R1 for $12 \mathrm{~h}$. The proliferation rates of the BA/F3 cells expressing NZW-IL-10R1 and G1146A-IL-10R1 were positively correlated with the IL-10 levels and were higher than those of the control group. The proliferation rate of the BA/F3 cells expressing G1146A-IL-10R1 was markedly higher than the proliferation rate of those expressing NZW-IL-10R1. However, the proliferation rate of the BA/F3 cells expressing WILD-IL-10R1 was negatively correlated with the concentration of IL-10 and lower compared with the control group (Fig. 2B).

IL-10 affects the expression of CD16/32 and LECAM-1 in BA/F3 cells expressing different types of IL-1ORI. Fig. 3A shows CD16/32 and LECAM-1 expression in BA/F3 cells expressing G1146A-IL-10R1, NZW-IL-10R1 and WILD-IL-10R1 following stimulation with IL-10. Compared with the BA/F3-expressing WILD-IL-10R1, CD16/32 expression on the BA/F3 cells expressing G1146A-IL-10R1 and NZW-IL-10R1 was decreased following stimulation with IL-10. The G1146A-IL-10R1 cell expression was decreased compared with cells expressing NZW-IL-10R1. Among the three cell types, the expression of CD16/32 was lower compared with that of the control (Fig. 3B). The expression of LECAM-1 in the BA/F3 expressing WILD-IL-10R1 was markedly decreased compared with the control and the other two groups. The expression of LECAM-1 in BA/F3 cells 
expressing G1146A-IL-10R1 was decreased compared with the control. However, the expression of LECAM-1 in BA/F3 cells expressing NZW-IL-10R1 was higher compared with the control (Fig. 3C).

Intracellular signal transduction of different types IL-10RI on $B A / F 3$ cells. Levels of the intracellular signal transduction proteins Jak1, Stat3 and Tyk2, which are associated with IL-10R1, in BA/F3 cells expressing G1146A-IL-10R1, NZW-IL-10R1 and WILD-IL-10R1 without IL-10 stimulation were identical (Fig. 4). Following stimulation with $10 \mathrm{ng} / \mathrm{ml} \mathrm{IL-10}$ for $15 \mathrm{~min}$, the levels of the intracellular signal transduction proteins Jak1, Stat3 and Tyk2 were also identical in BA/F3 cells expressing G1146A-IL-10R1, NZW-IL-10R1 and WILD-IL-10R1 (Fig. 5A). However, phosphorylated (p)-Jak1 and p-Stat3 levels in BA/F3 cells expressing G1146A-IL-10R1 and NZW-IL-10R1 were markedly lower compared with BA/F3 cells expressing WILD-IL-10R1 following IL-10 stimulation (Fig. 5B and C), while the levels of p-Tyk 2 in the three types of cells did not differ significantly (Fig. 5D).

\section{Discussion}

As a cy tokine with an inhibitory function in the immune reaction, IL-10 has an important role in immunoregulation (15). Treg and Breg are negatively regulated by IL-10 secretion. IL-10 is now recognized as a cytokine associated with SLE morbidity (16). IL-10 has the ability to inhibit B-cell proliferation and differentiation. These effects are closely associated with IL-10R1 and the distribution of the functional regions is located in the cytoplasm (12). A comparison of IL-10 in CD57BL/6, NZW and MRL mice indicated 18 base replacements, seven of which induce amino acid changes. The most notable change was in G1146A, which is located in the immune inhibition region and blocks the linkage with the signal transduction protein to interrupt the ability of immune inhibition. The proliferation rate of BA/F3 expressing WILD-IL-10R1 was observed to be negatively correlated with the time and dosage of stimulation with IL-10, and was consistently lower compared with BA/F3 cells without stimulation. These results indicated that the intact immune inhibition region may regulate the ability of the proliferation region effectively and inhibit proliferation to downregulate the number of $\mathrm{B}$ cells and decrease the output of antibodies. By contrast, the proliferation rate of BA/F3 expressing G1146A-IL-10R1 was positively correlated with the time and dosage of stimulation. The results indicated that base replacement caused a loss in the regulation of proliferation. The general effect of IL-10 on the proliferation rate of BA/F3 cells expressing NZW-IL-10R1 was similar to that of BA/F3 cells expressing G1146A-IL-10R1. However, the proliferation rate of BA/F3 cells expressing NZW-IL-10R1 markedly lower, which may be associated with intracellular signal transduction disorders induced by base displacements located elsewhere. It is known that, due to the base replacement in the immune inhibition region, IL-10R1 loses the intracellular signal transduction ability necessary for proliferation inhibition, which may impair the inhibition of B-cell proliferation (17). In SLE, B-cell functions cannot be inhibited and the output of antibodies cannot be downregulated, which increases with the onset and progression of SLE (18).

In the present study, which investigated cellular signal transduction, the levels of p-Jak1 and p-Stat 3 were markedly downregulated in BA/F3 cells expressing NZW-IL-10R1 and G1146A-IL-10R1 following IL-10 stimulation. Furthermore, the levels of p-Tyk2 were not different from those in the BA/F3 expressing WILD-IL-10R1. This indicated that the base replacement in the inhibition region of NZW-IL-10R1 induced the loss of the Jak1 phosphorylation site and Jak1 and Stat 3 could not be phosphorylated. The results also indicated that the inhibition ability of IL-10 proceeded via the Jak1-Stat3 pathway (19), but not the Ttk2 pathway (9). Activation of this pathway may restore the inhibition ability of IL-10R1 for the treatment of SLE.

As described above, the base replacement in the inhibition region of NZW-IL-10R1 induces a loss in Jak1-Stat3 signaling. NZW-IL-10R1 does not inhibit B-cell proliferation induced by the proliferation region of IL-10R 1 effectively and downregulates the expression of CD32 to reduce inhibition of B-cell activation. NZW-IL-10R1 does not readily inhibit the LECAM-1 expression to induce the B-cell migration to the inflammation sites, thus potentially triggering SLE. Previous studies have indicated that MRL mice exhibit a specific defect regarding B cell stimulation by IL-10 (20). In Fasplr mice, the antibody levels of B- and T-cell subgroups and the progression of lupus were similar to the non-defect mice (20). A previous study showed that in MRL/lpr mice into which T2-MZP activated by anti-CD40 in vitro was transferred, the symptoms of SLE were ameliorated (21). At present, the role of T cells in the pathogenesis and progression of SLE is being increasingly investigated. A previous study indicated that mouse $\mathrm{CD} 8^{+}$ $\mathrm{T}$ cells expressed IL-10R1 immediately following activation (22). Mouse $\mathrm{CD}^{+} \mathrm{T}$ cells were also observed to express IL-10R1 immediately following activation. These findings indicated that IL-10 may regulate the function of mouse $\mathrm{T}$ cells immediately following activation. This hypothesis, combined with the observations of the present study, require further investigation and indicate that NZW-IL-10R1 may be implicated in the pathogenesis and development of SLE and may present a novel therapeutic target.

\section{Acknowledgements}

This study was supported by the Natural Science Foundation of China (nos. 30571701 and 30600541 ).

\section{References}

1. Heinlen LD, McClain MT, Merrill J, et al: Clinical criteria for systemic lupus erythematosus precede diagnosis, and associated autoantibodies are present before clinical symptoms. Arthritis Rheum 56: 2344-2351, 2007.

2. Beebe AM, Cua DJ and de Waal Malefyt R: The role of interleukin-10 in autoimmune disease: systemic lupus erythematosus (SLE) and multiple sclerosis (MS). Cytokine Growth Factor Rev 13: 403-412, 2002.

3. Houssiau FA, Lefebvre C, Vanden Berghe M, et al: Serum interleukin 10 titers in systemic lupus erythematosus reflect disease activity. Lupus 4: 393-395, 1995.

4. Mongan AE, Ramdahin S and Warrington RJ: Interleukin-10 response abnormalities in systemic lupus erythematosus. Scand J Immunol 46: 406-412, 1997. 
5. Mok CC and Lau CS: Pathogenesis of systemic lupus erythematosus. J Clin Pathol 56: 481-490, 2003.

6. Sprengers D, Stoop JN, Binda RS, et al: Induction of regulatory T-cells and interleukin-10-producing cells in non-responders to pegylated interferon-alpha therapy for chronic hepatitis B. Antivir Ther 12: 1087-1096, 2007.

7. Ray A, Basu S, Williams CB, et al: A novel IL-10-independent regulatory role for B cells in suppressing autoimmunity by maintenance of regulatory T cells via GITR ligand. J Immunol 188: 3188-3198, 2012.

8. Jankovic D, Kullberg MC, Feng CG, et al: Conventional T-bet(+) Foxp3(-) Th1 cells are the major source of host-protective regulatory IL-10 during intracellular protozoan infection. J Exp Med 204: 273-283, 2007.

9. Liu Y, Wei SH, Ho AS, et al: Expression cloning and characterization of a human IL-10 receptor. J Immunol 152: 1821-1829, 1994.

10. Ho AS, Wei SH, Mui AL, et al: Functional regions of the mouse interleukin-10 receptor cytoplasmic domain. Mol Cell Biol 15: 5043-5053, 1995

11. Riley JK, Takeda K, Akira S, et al: Interleukin-10 receptor signaling through the JAK-STAT pathway. Requirement for two distinct receptor-derived signals for anti-inflammatory action. J Biol Chem 274: 16513-16521, 1999.

12. Moore KW, de Waal Malefyt R, Coffman RL, et al: Interleukin-10 and the interleukin-10 receptor. Annu Rev Immunol 19: 683-765, 2001.

13. Qi ZM, Wang J, Sun ZR, et al: Polymorphism of the mouse gene for the interleukin 10 receptor alpha chain (Il10ra) and its association with the autoimmune phenotype. Immunogenetics 57 : 697-702, 2005
14. Yin Z, Bahtiyar G, Zhang N, Liu L, Zhu P, Robert ME, McNiff J, Madaio MP and Craft J: IL-10 regulates murine lupus. J Immunol 169: 2148-2155, 2002.

15. Akdis CA and Blaser K: Mechanisms of interleukin-10-mediated immune suppression. Immunology 103: 131-136, 2001.

16. Zhou M, Ding L, Peng H, et al: Association of the interleukin-10 gene polymorphism $(-1082 \mathrm{~A} / \mathrm{G})$ with systemic lupus erythematosus: a meta-analysis. Lupus 22: 128-135, 2013.

17. Donnelly RP, Dickensheets H and Finbloom DS: The interleukin-10 signal transduction pathway and regulation of gene expression in mononuclear phagocytes. J Interferon Cytokine Res 19: 563-573, 1999

18. Peeva E, Venkatesh J, Michael D and Diamond B: Prolactin as a modulator of B cell function: implications for SLE. Biomed Pharmacother 58: 310-319, 2004.

19. Valencia-Pacheco G, Layseca-Espinosa E, Niño-Moreno P, et al: Expression and function of IL-10R in mononuclear cells from patients with systemic lupus erythematosus. Scand J Rheumatol 35: 368-378, 2006.

20. Teichmann LL, Kashgarian M, Weaver CT, et al: B cell-derived IL-10 does not regulate spontaneous systemic autoimmunity in MRL.Fas(lpr) mice. J Immunol 188: 678-685, 2012.

21. Blair PA, Chavez-Rueda KA, Evans JG, et al: Selective targeting of B cells with agonistic anti-CD40 is an efficacious strategy for the generation of induced regulatory T2-like B cells and for the suppression of lupus in MRL/lpr mice. J Immunol 182: 3492-3502, 2009.

22. Biswas PS, Pedicord V, Ploss A, et al: Pathogen-specific CD8 T Cell responses are directly inhibited by IL-10. J Immunol 179: 4520-4528, 2007. 